\title{
Practice and Research of Chemistry Experiment Development in Fire Command Specialty
}

\author{
Zhenqing Li \\ Kunming Fire Service Training School \\ 13518787687@139.com
}

Keywords: Fire commander; Chemistry experiment; Talent training

\begin{abstract}
Facing the severe challenge of modern firefighting work, fire commander is required to have higher comprehensive quality, especially the relevant chemical specialty, while Chemistry is an experiment-based discipline. Hence, Chemistry experiment teaching is particularly important in Chemistry teaching. Research and practice of Chemistry experiments development with fire characteristics is of great importance to achieve the fire command professional training objectives and promote teaching reform and curriculum construction.
\end{abstract}

\section{Introduction}

Fire commander after professional training can adapt to the modern needs of fire work, with comprehensive development in the moral, intellectual, physical and aesthetic fields, solid foundation, high-quality business and innovative spirit, capable of the first job of fire brigade management and fire and rescue command, mastering knowledge and ability of the fire business training, administrative law enforcement, political work in military grassroots and logistics management and other aspects. Chemistry knowledge, especially dangerous chemicals disaster accident rescue, is necessary In fire prevention and fire work. Chemistry experiments in Chemistry teaching has a strong practical and guiding property and is of great significance to cultivate excellent fire command talent.

\section{Necessity of Developing Chemistry Experiment in Fire Command}

Status and Function of Chemistry Experiment in the Training of Fire Command Professional Talents. In the daily fire work, the fire risk of material was determined and the appropriate measures of fire prevention and fire were taken through discovering the nature of chemical substances. In the rescue process of dangerous chemicals accident and disaster, the commander can select scientific, reasonable and safe disposal measures and methods to ensure the successful disposal according to the characteristics and reaction principle of different dangerous chemicals.In recent years, a large number of chemical substances are widely used in people's production and living, generally passing the whole process from the initial producers to the final consumer through the production, processing, transportation, storage and other procedures, in which each hazardous substance is likely to be affected by changes of friction, impact, shock, squeeze, mixing, temperature and humidity that can lead to leakage, diffusion, burning, explosion, causing property damage and casualties.Furthermore, chemical disaster accidents occur suddenly and are unpredictable. Hence, a slight negligence or improper disposal can easily lead to the death of the group of malignant consequences. At the same time, with the development and utilization of new fire extinguishing agents, fire retardant coatings, fire equipment and personal protective equipment, the rescue capacity of fire brigade has been greatly improved, and all these are inseparable from the development of chemistry. Facing the severe fire work situation, fire officers and soldiers undertake more difficult task, which requires the firefighting commander of the basic level firefighting team to have higher overall quality, especially the relevant chemical professional knowledge.

Guidance of Chemistry Experiment Development in Chemistry Learning. Chemistry is an experiment-based discipline, and the experiment plays a leading role in Chemistry teaching, and the 
improvement of Chemistry teaching quality is inseparable from Chemistry experiment. As the soul and charm of Chemistry, the experiment is an important way and method to cultivate and improve the students' abilities, helping students to form the concept of Chemistry, understand and consolidate the knowledge of Chemistry, improving the students' ability to observe and innovate, cultivating their earnest and serious scientific attitudes, as well as creative thinking ability.The content, type, and number of Chemistry experiment are determined clearly according to Chemistrycharacteristics, students' learning law and cultivate characteristics and so on. Each experiment is divided into five parts of purpose, principle, instrument, procedureand precautions. The effects of Chemistry experiments are mainly as follows: First of all, on the basis of experiments, teachers identify the purpose and training objectives, prepare experiment such as the reaction principle and experimental drugs. Secondly, the students preview the experimental contentindependentlyto lay the knowledge base for Chemistry experiments. Finally, the key to the success of the experimentis to guide students the "experimental steps" and "precautions" and other content of the experiment, achieving the purpose of the experiment through analyzingand summarizing the experimental phenomenon and result (successor failure), and writing the experimental report.

Effects of IrregularChemistry Experiment on the Quality of Chemical Teaching in fire Command Specialty. IrregularChemistry experiment refers to that both the use of the teaching materials and the experimental content arrangement (not according to the unified syllabus and the teaching plan, the teachers carry out the Chemistry experiment in their own way) are random, as well as the experimental site, the experiment operated by studentsis extensive, formalized and just demonstrated by teachers, while demonstration experiment turns to be electronic. The effect of irregularChemistry experiment on the quality of firefighting command professional training is as follows: (1) The objective of chemistry curriculum is difficult to realize. (2) fire education features can not be reflected. (3) students lose interest in learning chemistry, and even raise aversion to chemistry learning. (4) students' ability to observe and operate is hard to improve. (5) the student experiment becomes demonstration experiment, while the demonstration experiment becomes experiment of watching image, and the students can not experience the success and failure of Chemistry experiments, not convenient to cultivate students' correct attitude of emotional values. (6) due to the absence of exploratory experiments, students can not cultivate creative and independent thinking ability to solve problems. (7) the teachers' positive initiative has also been affected, as well as their scientific research ability, teaching ability and teaching level can not be effectively improved.

\section{Objectives of Chemistry Experiment Development in Fire Command Specialty}

Making the Improvement of Combat Capability and Innovation Ability as the Core of Teaching. Chinese Eleventh Five-Year Plan of National Education Development points out thatthe focus of the development of higher education is to improve the quality, train students' innovative spirit and innovative thinking, enhance students' practical ability, creativity and employability.To conductteaching reform of higher education undergraduate teaching quality, colleges and universities should try to increase teaching investment, improve teaching conditions, especially experimental practice conditions to promote a new round of curriculum and teaching content reform, continue to Improve the teaching methods and means to explore innovative talents training mode, advocate research learning and research activities of undergraduate students, establish long-term mechanism of students going to enterprise research institutions and scientific research institutes. Public Security Department of Fire has also put forward the fire education philosophy that fire education should be oriented to the basic unit and the first line of fire.

"Big education and training system" is involved in not only extensive educational staff but also the change of training content, training methods, and training concept, which will have far-reaching influences to the fire command professional teaching philosophy and curriculum system settings. It is the central link to deepen the teaching reform and improve the quality of education by strengthening the training of students' actual ability and innovation ability. In order to meet the needs of the reform of the 
military colleges and universities and the requirements to talents of the basic units, it is necessary to change the concept and make improvement of the basic fire commander combat capability and innovation ability as the core of the Chemistry experimentteaching, according to the reality of the comprehensive construction of fire brigade and the practical fire rescue.

Determination Objectives of Experimental Teaching with Fire Characteristics. Taylor sums up the sources of the course objectives into three areas as follows: student which stands for the research oflearners, society whichstands for the study of off-campus contemporary life, and subject standing for study by subject experts. The course objectives are obtained from suggested objectives resulted byscreening of these three sources The analysis and selectionprocess of course objective can be shown in Fig. 1.

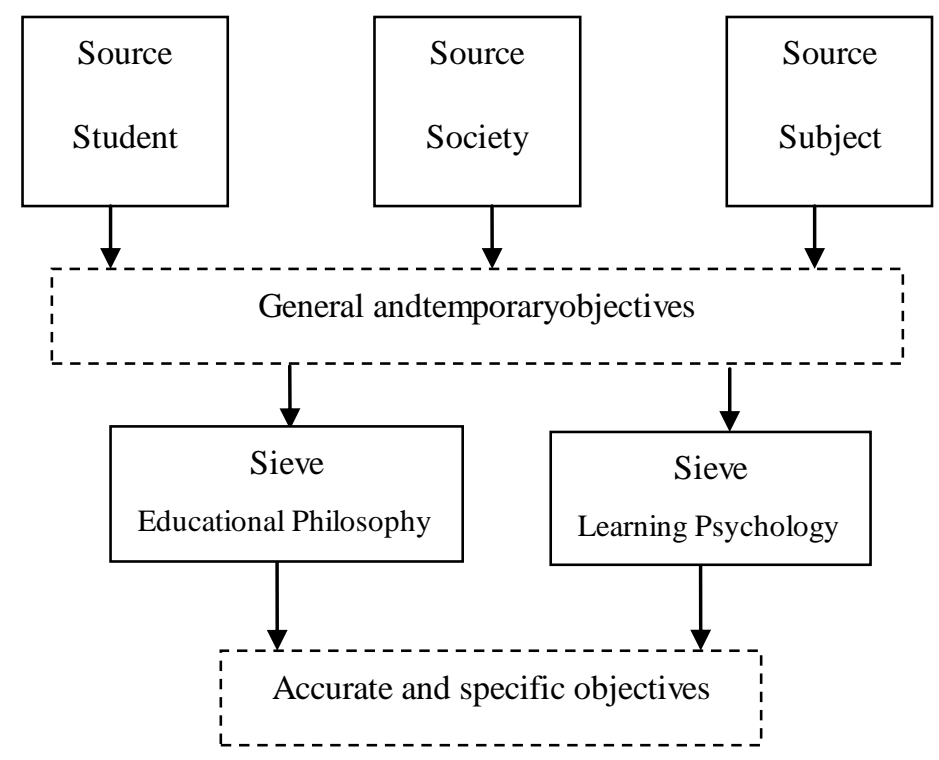

Figure 1. Finite Taylor's analysis and selection process of course objective

The course of fire chemistry must focus on the objective of fire command professional training. Through the course teaching of Chemistry experiment, the students can understand the basic knowledge of chemical theory, master the basic experimental operation methods and skills, as well as the combustion theory, fire theory and the hazardous chemicals and physical and chemical properties, and the knowledge applied to fire prevention, fire, hazardous chemical disaster accident disposal, so that students have a strong practical ability, innovation, teamwork awareness and a solid foundation for the follow-up courses and their future development. In order to realize the objective of training firefighting command professional personnel and fully embody the purpose of Chemistry experiment curriculum with fire characteristics, the development of Chemistry experiment should follow four basic principles: First, the characteristics should be highlighted to train the fire command personnel suitable for the development of firefighting work. The second is to develop the student-oriented, pursuit school-based materials to the greatest possibility to serve the development needs of students, strengthen the basic experiment, and highlight the design experiment and exploratory experiment. The third is to develop moderately advanced, and the development of experimental materials from the future needs with foresight and forward-looking, reflecting educational philosophy of "stricter, prior to, and higher than the troops". Fourth, long-term planning is necessary to always keep the experimental development and construction as a constant reflection, Improved sustainable development process.

\section{Development of Experimental Teaching Content of Fire Command Specialty}

Chemistry experiments having different classification methods, according to the different experimental subjects, can be divided into demonstration experiments (subject is the teacher) and student experiments (subject is the student). According to the experimental content, the relationship ofquality and quantity they can be divided into qualitative experiment and quantitative 
experiment.According different effects of the experimental study on the masteringof chemical basic knowledge and Chemistry experimental skills, they can be divided into the basic operation experiment, experiment of the compound nature and preparatory experiment, experiment of the formation of chemical concepts and basic theory, experiment contacting with social production and daily life, and experiments to solve chemical or professional problems and so on.

Experiments about Combustion Conditions and Fire Principle. Burning, commonly known as "fire", is the violent redox reaction between combustible substance and oxidant, usually accompanied by flame, light and smoke phenomenon. Combustion has three characteristics, that is, chemical reactions, heat and light, and three basic conditions: combustible material, auxiliary combustible material (oxidant) and ignition source (energy). Only when these three conditions interact can combustion be produced. It is known that the firefighting is the process of destroying the combustion conditions to terminate the combustion chain reaction (ie, the disappearance of free radicals). The basic principles are summarized as follows: cooling, choking, isolation and chemical suppression.

The Properties Experiment of Common Dangerous Chemicals. Chemicals are a general term for a variety of chemical substances (chemical substances). The definition of International Labour Organization (ILO) for chemicals is that chemical refers to a variety of chemical elements, a compound consisting of several elements and their mixture, whether natural or man-made. Chemicals are flammable, explosive, toxic, harmful, corrosive, radioactive and with other characteristics, which can cause injury or damage to personnel, facilities and environment.There are many kinds of dangerous chemicals with complex components, which are dangerous, such as inflammable, explosive, toxic, corrosive and so on. Whether it is in production, storage, transportation or operation of any part of the use, as long as the slightest mistake may lead to accident. And when it occurs, the disaster has the characteristics of fast andwide spread, difficult disposal and decontamination. Furthermore, it can also cause secondary disasters since many hazardous chemicals gathering many harmful characteristics in one,bringinga great challenge to of hazardous chemicals to the emergency rescue work. The contents of Chemistry experiment in fire control specialty should include the dangerous and hazardous experiments of various types of dangerous chemicals in Classification and Name of Dangerous Goods (GB6944-2012).In the content selection, we should start from the typical case of dangerous chemical accidents, combined with the content of chemistry courses, and select the representative dangerous chemicals to carry out the experiment. The main purpose of the experiment is to enable the students to consolidate the basic knowledge of chemistry through experiments, understand and grasp the nature of dangerous chemicals, hazardous characteristics and disposal measures.

Comprehensive Experiment. Comprehensive experiment refers to the content of the course involving the comprehensive knowledge of the course or the relevant knowledge of the curriculum experiment.The purpose of setting up a comprehensive Chemistry experiment is to train the students' experimental skills in a comprehensive way, so as to cultivate the students' ability of comprehensive analysis, experimental ability, data processing and the ability to access data. Comprehensive experiment has four characteristics of the complexity of the experimental content, the diversity of the experimental methods, the diversity of experimental methods and the comprehensive nature of personnel training.

Designing Experiment. Designing experiment is a kind of exploratory experiment which is designed and implemented by the students and combined with or independent to the teaching of the course, whose focus is on training students' ability to solve practical problems independently, innovation and organizational management capabilities. To design the experiment, it is suggested that the teachers should put forward the purpose of the experiment according to the teaching requirements, the laboratory devicesprovided including drugs, reagents and other experimental conditions, while students use the basic knowledge, basic principles and experimental skills to put forward the concrete scheme of the experiment, draw up the experimental procedure, select the instrument and equipment (or the device, the medicine, the reagent, etc.), and also completethe experiment independently, such as operation, the experimental data record, drawing the chart, and analyzing the experimental result 
and etc.The process of the experiment should give full play to the initiative of the students, guide students to innovative thinking, reflecting the spirit of Science.

\section{Conclusions}

The research and development of Chemistry experimentof fire command professionalis a long and complex process of repeated practice, feedback, evaluation and improvement, which fully reflects the position and function of Chemistry experiment in Chemistry teaching, really presenting the chemistry education with image, characteristic, effect and charm.

\section{References}

[1] Ministry of Public Security Fire Department. China Fire Yearbook [M]. Beijing: China Personnel Publishing House, 2009.7.410.

[2] Wang Xitong. Chemistry experimental teaching research [M]. Beijing: Higher Education Press, 1990.3.1-6

[3] Wang Yonghong, Li Chunhong. Overview of Foreign Fire Education [J] Global Fire, 2006,8 (12): 36-38.

[4] Chemistry Textbook Writing Groupof Vocational College. Inorganic Chemistry [M]. Beijing: Higher Education Press, 2002.

[5] Higher Vocational College Chemistry Textbook Writing Group. Organic Chemistry [M]. Beijing: Higher Education Press, 2002.

[6] Wu Jiang. University of basic Chemistry experiment [M]. Beijing: Chemical Industry Press, 2005.8

[7] Yuan Zhenuo. ContemporaryPedagoge [M]. Beijing: Education and Science Press, 2004.6.131.

[8] Liu Zhixin. Chemistry curriculum theory [M]. Guangxi: Guangxi Education Press, 1996.11.31-39.

[9] People's Republic of China Ministry of Education. National Education Development Eleventh Five-Year Plan Outline [M]. Beijing: People's Education Press, 2007.6

[10] Gao Hongze. Analysis of the status of chemistry in fire [J]. Journal of the Armed Police Force Academy, 2007, 18 (3): 94-96 